- The creation of a freely available online resource

- 2204 people registered for Listening and Sharing sessions

- 19 sessions held in 6 languages

- 45 countries

- 18 disciplines attended

Feedback forms were sent after the $24 \mathrm{~h}$ event and responses analysed $(n=21)$. Responses covered 5 continents and 12 countries (limited by only being available in English and sent out some time after the event).

Conclusions Listening and Sharing sessions provided a useful format to rapidly share issues, ideas, and good practice across disciplines and around the globe. This format proved easy for all to engage with and may be useful as a future tool for rapid communication and sharing of knowledge, experience, and skills. Listening and Sharing sessions may be particularly useful where acquisition and transfer of knowledge is time critical. The Global Task Force was very active during the first phase of the pandemic but not during the second wave which may be an indication of people being better prepared.

\section{KNOWLEDGE AND AWARENESS OF MATERNAL CHRONIC HEPATITIS CARRIERS TO PERINATAL ANTIVIRAL USE IN PREVENTION OF MOTHER-TO-CHILD HEPATITIS B VIRUS TRANSMISSION}

Jia Ming Low, Guan-Huei Lee. Singapore

\subsection{6/bmjpo-2021-RCPCH.160}

Background Antiviral treatment is recommended to reduce the vertical transmission in women with high viral load for mother-to child-transmission (MTCT). However, most women decline treatment.

Objectives We aimed to determine the knowledge and awareness of maternal chronic hepatitis B carriers to antiviral use.

Methods A cross sectional study was carried out in a tertiary hospital in National University Hospital of Singapore. Maternal hepatitis B carriers with children less than 5 years old were surveyed on attitudes and knowledge on hepatitis B virus as well as what their reasons were for taking/not taking antivirals.

Results Fifty-seven were surveyed; 50.9\% had a degree, $64.9 \%$ worked full time, $47.4 \%$ had 1 child or more. Most were risk averse. Many, $78.9 \%$ were not aware of the role of antivirals to reduce MTCT. Only $21.1 \%$ knew the risk of vertical transmission with/without antiviral. Reasons for not taking antivirals were attributed to a lack of knowledge. Many, $87.7 \%$ are willing to take antiviral therapy during their pregnancy to reduce MTCT from $10 \%$ to $1 \%$ and $45.6 \%$ willing to pay at least $\mathrm{S} \$ 2$ to $\mathrm{S} \$ 2.90$ daily over 12 weeks for it.

Conclusions It is the practitioner's role to update and improve the quality of education programmes that target women of childbearing age about the benefits of antiviral use to reduce MTCT in mothers with high hepatitis B viral load. Additionally, from a health system perspective, providing subsidies for antiviral treatment to ease the financial concerns of parents, is likely to reduce long-term expenses generated by chronic hepatitis B virus complications, thus being more costeffective.

\section{PREMFIRST HOUR: ADAPTING THE GOLDEN HOUR TO IMPROVE OUTCOMES}

Janlie Banas, Alvin Chang, Bin Huey Quek, Juin Yee Kong, Rajammal Kaliappan, Nordiana Sulaiman. Singapore

\subsection{6/bmjpo-2021-RCPCH.161}

Background Despite existing guidelines in the initial management of preterm infants, KK Women's and Children's Hospital Neonatal Unit's 'golden' first hour stabilization rate was only twenty-eight percent (28\%) in November 2018. Variations in practices post-resuscitation were observed and this had resulted in non-attainment of stabilization of our infants within the golden hour.

Objectives To increase the number of infants born preterm less than thirty-three (33) weeks gestation stabilized within the golden hour by thirty percent (30\%) in six (6) months.

Methods Baseline stabilization time data was collected from eighteen (18) preterm infants less than thirty-three (33) weeks gestation over a one-month period. A questionnaire-based survey was conducted among one hundred six (106) staff to assess their concept of golden hour stabilization, and to recognize the challenges encountered on the ground. We found that only fifty-eight percent (58\%) were convinced that one hour is the ideal stabilization time and that the lack of manpower was a major challenge to the achievement of golden hour stabilization. We performed a root cause analysis and mapped out the golden hour stabilization process. The key areas of concern leading to non-attainment of the golden hour goals were not achieving normothermia on admission, placement of intravenous glucose or parenteral nutrition and blood gas sampling. Interventions to address the key areas of concern in the golden hour stabilization process were put in place. These included: (1) Standardizing work processes with time-specific, well-defined roles for all healthcare staff involved in golden hour stabilization. (2) Use of a countdown timer to improve situational awareness during the first hour of stabilization. (3) Improving multi-disciplinary teamwork by encouraging communication and recognizing good team dynamics. (4) Roadshows and educational sessions among neonatal doctors and nurses to promote engagement. (5) Revision of admission criteria to the Neonatal Intensive care Unit (NICU). (6) Continual update of the department's 'Prevention of Hypothermia Workflow'. All interventions were subjected to multiple PlanDo-Study-Act (PDSA) cycles for further refinement. The stabilization time was then tracked over time and compared with baseline.

Results The rates of infants stabilized within the golden hour improved from $28 \%$ to $97 \%$ after the interventions. Likewise, the mean and standard deviation stabilization time improved from 88 to 45 minutes and 43 to 13 minutes respectively.

Conclusions Streamlining practices and embarking on initiatives addressing hypothermia resulted in more infants stabilized within the golden hour. This had reduced variability in stabilization time, indicating a reduction in varied clinical practices in the unit. 